\title{
Perioperative management of complex skull base surgery: the anesthesiologist's point of view
}

\author{
W. Scott Jellish, M.D., Ph.D., John Murdoch, M.D., And John P. Leonetti, M.D. \\ Departments of Anesthesiology and Otolaryngology Head and Neck Surgery, Loyola University \\ Medical Center, Maywood, Illinois
}

\begin{abstract}
The anesthetic management of complex skull base surgical procedures provides unique problems and concerns for the neuroanesthesiologist. Positioning to access the skull base could put the patient at risk for peripheral nerve injury and some of the positions may increase the risk for air emboli. In addition, tumor pathology and involvement with vital structures could increase the chances for substantive blood loss, destruction of associated nerves or vessels, and may require temporary occlusion of the carotid artery necessitating intraoperative neuroprotection. Neurophysiological monitors may also be used to safeguard nerve function and anesthetic techniques must be adjusted to accommodate their use. Finally, postoperative morbidity may be affected by surgical approach to the skull base and the anesthesiologist should be aware of which approach may produce a greater incidence of pain, nausea, and vomiting in the postoperative period.

The authors discuss the anesthetic concerns and management for complex cranial base surgery. Different approaches will be discussed and comparisons of perioperative parameters between these approaches will be made with data provided by retrospective chart review of more than 600 skull base procedures performed at the authors' institution over the last 10 years. This information should help guide decision making concerning anesthetic management for these skull base procedures.
\end{abstract}

KEY WORDS • anesthesia • skull base surgery • air emboli • surgical approach

The surgical approach to the skull base has evolved tremendously in a relatively short period of time. As the field of skull base and craniofacial surgery has evolved, new approaches to the skull base have been described. With these new surgical approaches, as well as technical advances in intraoperative monitoring and anesthetic care, the anesthesiologist has assumed more responsibility for the perioperative management of the patient. Lesions that were considered inoperable because of location and risk to other juxtaposed vital structures are in many instances curable and can be surgically corrected without long-term morbidity for the patient.

For the anesthesiologist, some of the knowledge and problems are new, while others have not changed much from the past. Initially, the challenges that come with anesthetizing a patient for any surgical procedure begin with an understanding of the patient's basic physical and mental health as well as the primary type of tumor and risks involved with inducing general anesthesia.

Abbreviations used in this paper: $\mathrm{CA}=$ carotid artery; $\mathrm{EEG}=$ electroencephalography; EMG = electromyography; SSEPs = somatosensory evoked potential; TEE $=$ transesophageal echocardiography; VAE = venous air embolism.
Most skull base neoplasms are benign slow-growing tumors and patients do not present with early symptoms. An exception to this is the neurosecretory glomus tumors which may manifest nonspecific systemic symptoms early in the disease process. It is also important for the anesthesiologist to know the primary type of tumor, its location within the skull base, the proximity of vital structures, and which skull base approach is to be used. This knowledge will be helpful in preparing for proper patient positioning and assessment of potential or general neurological dysfunction. We will first review the surgical approaches to the skull base and then discuss specific concerns the anesthesia consultant faces in the management of these complex skull base surgeries.

\section{SURGICAL APPROACHES}

The surgical approaches to the skull base have been developed, refined, modified, and in some cases, combined to provide the best possible exposure with the least amount of associated morbidity for the patient. Simply put, they are transfacial, transtemporal, and occipital approaches (that is, bone routes) to the neoplasm (Table 1). Anesthetic management for these complex skull base approaches has some important concerns and risks that in- 
TABLE 1

Approaches to skull base

\begin{tabular}{|c|c|}
\hline Approach & Description \\
\hline \multicolumn{2}{|l|}{ classic } \\
\hline craniofacial & $\begin{array}{l}\text { anterior cranial fossa; extradural lat rhinotomy; ethmoidectomy; sphenoidectomy; intradural, unilat, or bifrontal craniotomy; } \\
\text { elevation or resection of frontal lobe; osteotomy of skull base }\end{array}$ \\
\hline orbitocranial & anterior cranial fossa; supraorbital ridge osteotomy \& elevation of frontal and/or temporal lobes \\
\hline infratemporal & $\begin{array}{l}\text { middle cranial fossa; parapharyngeal space; infratemporal \& midline skull base, w/ or w/o disarticulation of mandible; } \\
\text { parotidectomy; craniotomy, decompression \&/or mobilization of facial nerve or pertrous CA }\end{array}$ \\
\hline $\begin{array}{l}\text { suboccipital } \\
\quad(\text { transcondylar) }\end{array}$ & $\begin{array}{l}\text { posterior cranial fossa; midline skull base; occipital condylectomy; mastoidectomy; resection of C1-3 vertebral body; de- } \\
\text { compression of vertebral artery }\end{array}$ \\
\hline \multicolumn{2}{|r|}{ 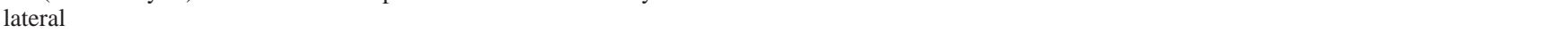 } \\
\hline retrosigmoid & posterior cranial fossa or midline skull base mastoidectomy; decompression of sigmoid sinus \&/or facial nerve \\
\hline translabyrinthine & $\begin{array}{l}\text { posterior cranial fossa; jugular foramen or midline skull base; labyrinthectomy; decompression of facial nerve \&/or } \\
\text { petrous CA }\end{array}$ \\
\hline orbitocranial zygomatic & $\begin{array}{l}\text { middle cranial fossa; (cavernous sinus \& CA, clivus basilar artery) osteotomy of zygoma; craniotomy; extra- or intradural } \\
\text { elevation of temporal lobe }\end{array}$ \\
\hline
\end{tabular}

fluence patient outcome. The following represent some of the primary concerns of the anesthesiologist when considering the intraoperative management of a patient undergoing a skull base surgical resection.

\section{AIRWAY}

Airway management in patients undergoing skull base surgery is an important consideration. In most scenarios, the airway is not shared with the surgeons and the concerns of the anesthesiologist center on airway anatomy and the possibility of a difficult intubation. With the transoral, transmandibular, or transmaxillary approaches to the skull base, the airway is shared with the surgeon and a decision must be made to determine whether oral tracheal intubation will facilitate the surgical approach or if the patient will require a tracheostomy. Many times the decision to intubate or perform a tracheostomy is made after discussions with the surgeon to obtain an estimate of the amount of upper airway edema that may occur postoperatively. If excessive edema is expected, a tracheostomy should be placed to assure a controlled and stable airway in the postoperative period. Thus airway management for these particular approaches will be predicated on postoperative airway edema and the need for airway protection.

\section{PATIENT POSITIONING}

Operative positioning has been found to influence complication rates. Patient positioning for skull base tumor resection can be complex and the procedures may be prolonged. Bone protuberances should be padded with foam, and in some instances the elbows must be wrapped completely to avoid ulnar neuropathy from pressure point contact at the ulnar groove. Metal edges should be isolated from the patient and abnormal flexion or extension of extremities checked to avoid diminished perfusion or nerve plexus injury. Padding should also be placed under the heels and buttock area. A pillow should be placed behind the knees to reduce tension on the back, especially during procedures in which the patient is supine for a long duration. Meticulous care with positioning is important because it is extremely difficult to reposition a patient once the surgery begins.

It is well recognized that head-above-heart positioning (that is, sitting and "park bench" positions) offers better surgical exposure and the potential advantages of improved hemostasis $;{ }^{26}$ however, as has been well described, these "head-elevated" positions carry with them a higher incidence of VAE and pneumocephalus. ${ }^{34}$ Open sinuses, noncollapsable bonelike sinuses and large veins like the jugular can contribute to a heightened risk for VAE. Also, increased risks of quadriplegia, paraplegia, peripheral nerve injuries, and facial/glossal edema have been reported when patients are placed in the sitting position. ${ }^{25} \mathrm{Ve}-$ nous air embolism can occur in all types of tumor surgery and should be a consideration when the surgical field is higher than the heart. In addition, many neurosurgical patients with tumor are hypovolemic, which reduces central venous pressure and further increases the chances for air embolization. Convexity meningiomas, especially if there is bone involvement, should be considered for VAE monitoring. The bone has been noted to be the source of VAE in $43 \%$ of all sitting craniotomies. ${ }^{16}$ The incidence of neurosurgical skull base VAE in any head-elevated position has been noted to be $28 \%$ for sitting craniotomies compared with $5 \%$ for supine and prone positions. ${ }^{7}$ Because many skull base resections involve the jugular vein and cavernous or sigmoid sinuses, and considering that some positioning requires slight head elevation, the risk of passive air entrainment is increased. With the transtemporal and transcochlear approaches to the skull base, the patient is supine and the surgical field is neutral to the heart. With these approaches, we do not use prophylactic measures to monitor and protect against VAE.

If the procedure does warrant monitoring for VAE, we prefer the use of the precordial Doppler (Versitone Model D8; Medasonics, Fremont CA). Although many investigators have pointed to the increased sensitivity of TEE for detection of VAE, ${ }^{10}$ we believe this increased sensitivity produces too many false positives, reducing its specificity as a monitor for air entrainment. In addition, TEE is expensive and requires trained personnel to be continually available for interpretation. The size of the probe implies some potential for injury of the esophagus or larynx, and 
there are several reports in which esophageal rupture ${ }^{13}$ and recurrent laryngeal nerve injury ${ }^{27}$ with the use of TEE are described. The unusual positioning used for some skull base approaches and the overall operative duration of some of these cases might increase the risk of airway trauma or nerve injury with TEE. An angiographic catheter (Swan Ganz Angiography Catheter; Baxter Healthcare Corp., Irvine, CA) is used for aspiration of air from the right atrium should it occur. This multiorifice catheter is placed in a similar fashion to that of a Swan Ganz catheter and requires the use of a small pilot balloon at the tip, which allows the catheter to be easily floated into the right ventricle. The single port is connected to a transducer for detection of the right ventricular waveform. Once the waveform is observed, the catheter is withdrawn 1 to $2 \mathrm{~cm}$ until the trace disappears. This places the catheter in the proper position.

Lateral supine positioning of the patient for the translabyrinthine, retrosigmoid, and orbitocranial zygomatic approaches has reduced the risk of VAE but has been implicated in peripheral nerve injury. ${ }^{39}$ The most persistent complaint of nerve injury after lateral approaches to the skull base, aside from cranial nerve injury, is that of the brachial plexus. Closed claims analysis of brachial plexus injury note that they are mostly associated with general anesthesia, with $10 \%$ related to patient positioning (head down, malpositioning of the arms, or sustained neck extension). ${ }^{5}$ Retrospective findings obtained from our own surgical database show a $9 \%$ incidence of brachial plexopathy, with $82 \%$ of these injuries occurring in patients undergoing a lateral approach to the skull base. Sixty-one percent of these injuries were ipsilateral to the surgical field and $18 \%$ of these patients had bilateral symptoms. In most cases, these symptoms resolved within 2 to 3 days. Because a large proportion of these injuries occurred on the operative side, the most likely cause was traction and stretching of the brachial plexus during patient positioning for the lateral approach. Rotating the head contralaterally away from the surgical field, elevating the ipsilateral shoulder, and providing downward traction on the arm stretches the brachial plexus. The anesthetic care team should be focused on these positioning problems and protect the patient from overextension or flexion injuries that can occur.

\section{BLOOD LOSS}

More studies are needed to discern the morbidity rates resulting from blood loss during complex skull base surgeries. Given the absence of well-documented studies in which this comorbid risk is compared with a given surgical approach, some generalizations can be made. In cases in which marked cerebral edema is present or the tumor is particularly large, there is a tendency to restrict fluid to reduce brain volume. This rationale is flawed, given the severe physiological stress that develops in the patient with hypovolemia and the minimal benefit in brain water reduction that is produced $(8 \%$ decrease in body water produced a $1 \%$ decrease in brain fluid volume). Extreme fluid restriction can result in hypervolemia, hypotension, and possible ischemia from hypoperfusion. The overall goal of the anesthesiologist is to maintain normal intravas- cular volume. Our practice is to replace obligatory losses slowly and supplement patients who have recently undergone angiographic studies because of the diuresis that occurs after use of radiographic imaging solutions. Isoosmolar crystalloid is sufficient to replace urine output and insensible losses on a one-to-one basis. As a general rule hyposmolar solutions and those containing dextrose are avoided.

Lesion characteristics such as size, location, and histological type should be considered in planning for blood loss. Highly vascular neoplasms, such as meningiomas and glomus tumors, can be associated with substantial blood loss. Tumor involvement and extension into cranial sinuses and blood vessels increases the likelihood of more blood loss.

Meningiomas have been shown to produce a tissue plasminogen activator that leads to significant changes in fibrinolytic parameters and increased blood loss during tumor resections. ${ }^{30}$ Excessive fibrinolysis can induce a local hemorrhagic diathesis during surgery. Some investigators have noted the development of a disseminated intravascular coagulopathy during primary brain tumor resections. This could develop from tumor plasminogen activators or destruction of the blood-brain barrier leading to vascular liberation of factors activating hemolysis. In addition, molecules on the tumor surface could activate the coagulation cascade through nonspecific tissue thromboplastin. ${ }^{9}$

Strategies implemented to guard against significant intraoperative blood loss include hypotensive anesthetic techniques, surgical control of major vessels, and preoperative tumor embolization. Taken as one or together, these techniques can contribute to diminishing the risk of substantial intraoperative blood loss for a given patient. The surgical approach could also dictate the intraprocedure blood loss. Retrospective analysis of our surgical database reveals that suboccipital approaches to the skull base produced the highest estimated blood loss $(1467 \pm 70 \mathrm{ml})$ whereas patients undergoing a retrosigmoid craniotomy had the smallest average blood loss $(311 \pm 49 \mathrm{ml})$. In general, patients undergoing craniofacial, orbitocranial, infratemporal, and suboccipital approaches to the skull base had higher mean blood loss $(1218 \pm 148 \mathrm{ml})$ compared with those undergoing retrosigmoid and translabyrinth craniotomies $(457 \pm 37 \mathrm{ml})$. Compared with those undergoing suboccipital, orbitocranial, and craniofacial skull base approaches, those patients undergoing lateral skull base approaches required less crystalloid (3304 \pm 99 versus $4559 \pm 264)$ less colloid (591 \pm 46 versus $1343 \pm 70)$ and less total fluid $(3890 \pm 117$ versus $5901 \pm 380)$.

Some lesions may require resection through a specific surgical approach to achieve success; however, if surgical success can be achieved by performing different craniotomies, the surgeon should choose the approach that has the lowest comorbid risks. Based on our observation, it appears the risk of bleeding is much less with the lateral approaches to the skull base compared with the more classic approaches.

\section{PERIOPERATIVE NEUROPROTECTION}

Another concern of the anesthesiologist during skull base tumor resection is management of the CA. Some 
tumors either surround portions of major vessels or directly invade the external capsule. Resection of these tumors may necessitate complete occlusion or sacrifice of the involved artery or vein. If temporary occlusion of the CA is contemplated, the anesthesiologist should be informed early so that a plan may be developed to protect the brain intraoperatively. Some of the options that could be used to reduce neurological injury include support of blood pressure to increase perfusion through the circle of Willis and collateral circulation. ${ }^{35}$ Infusions of vasoactive agents such as neosynephrine or increasing blood pressure with crystalloid therapy could increase perfusion while simultaneously reducing viscosity to improve blood flow to the ischemic area. ${ }^{32}$ Blood pressure is targeted at 10 to $15 \%$ above awake-patient values whereas the hematocrit level is preferentially titrated to approximately $30 \%$.

Blood glucose concentration is also important during episodes of ischemia. It is postulated that hyperglycemia, in the presence of hypoxia, will increase intracellular acidosis. ${ }^{37}$ Insulin has a direct protective effect on ischemic neural tissue. Not only does it reduce blood glucose levels, it stimulates $\mathrm{Na}^{+} \mathrm{K}^{+}$adenosine triphosphatase, which enhances $\mathrm{Na}^{+}$extrusion with $\mathrm{K}^{+}$entry into the cell. Insulin also protects mitochondria and modulates synaptic transmission. Insulin and glucose infusions should be used to titrate the patient to a near-normoglycemic level. ${ }^{33} \mathrm{Ad}-$ ministration of steroids, although necessary to reduce cerebral edema, may be detrimental during periods of incomplete forebrain ischemia because of the associated hyperglycemia. Again, insulin infusions should be used to reduce blood glucose to more normoglycemic levels if carotid artery occlusion is anticipated. ${ }^{36}$

Hypothermia can also be used to provide neuroprotection during episodes of diminished cerebral blood flow. Prior to the anticipated CA occlusion, the patient's body temperature can be lowered by passive convective cooling in the operating room. Temperatures can be easily reduced to $34^{\circ} \mathrm{C}$, which has been found to decrease cerebral metabolism by 10 or $15 \%$ from normothermic levels. ${ }^{38}$ It has also been demonstrated in animal studies that significant reductions in excitatory amino acid release have occurred after forebrain ischemia with moderate hypothermia. ${ }^{3}$ Transmembrane ion flux is also reduced and basal energy expenditure is diminished providing a neuron-sparing effect.

Other protective measures available to the anesthesiologist include the use of hypnotic agents to reduce cerebral metabolic activity. Barbiturates are the most extensively studied cerebroprotectants. They reduce cerebral metabolism in a dose-dependent fashion, with reductions as high as $50 \%$ at levels titrated to EEG electrical silence. Barbiturates are also thought to enhance gamma aminobutyric acid activity and antagonize the $N$-methyl-D-aspartate receptor, which reduces ischemic excitotoxicity. ${ }^{20}$ Prior to intraoperative CA occlusion, a thiopental infusion can be initiated and titrated to a 2- to 5- second EEG burst suppression. Without the benefit of EEG, under or over titration of drug could occur. This may lead to a reduced neuroprotective effect or severe cardiovascular suppression, respectively.

Propofol, like other barbiturates, will induce burst suppression in a dose-dependent fashion. This drug has the potential to be as beneficial as thiopental. Furthermore it is metabolized quickly and therefore does not accumulate, providing a more predictable wake-up time for the patient. Although propofol has been shown to be of some benefit, results of animal studies have been inconclusive when compared with those for thiopental. ${ }^{40}$ Etomidate, an imadazole derivative, was a hypnotic agent used for neuroprotection in the late 1980s and early 1990s. Its cerebral metabolic profile is similar to thiopental and it produces a dose-dependent reduction in cerebral metabolic rate. The shorter half life and associated hemodynamic stability made etomidate a superior drug compared with thiopental for neuroprotection. Recent studies have demonstrated, however, that etomidate administration prior to cerebral ischemia produces a $30 \%$ reduction in $\mathrm{PaO}$ levels and a $23 \%$ increase in $\mathrm{PaCO}_{2}$ concentrations in the cerebral cortex. ${ }^{8}$ These changes were thought to be due to etomidateassociated vasoconstriction and a reduction in cerebral blood flow prior to a drop in cerebral metabolic rate. Presently, etomidate is not used by our group for neuroprotection.

Volatile anesthetics can also be used to reduce cerebral metabolism but their universal ability to dilate the arterioles and reduce blood pressure make them a less than optimal choice when attempting to maintain cerebral perfusion to ischemic areas. Other agents such as $N$-methylD-aspartate receptor antagonists, $\mathrm{CA}^{++}$channel blockers, free radical scavengers, and ion transport inhibitors have been administered in human trial studies with minimal success. The local anesthetic lidocaine may provide some added benefit through its ability to block the $\mathrm{Na}^{+}$channel, reduce $\mathrm{Na}^{+} \mathrm{K}^{+}$transmembrane flux, and decrease basal energy expenditures. ${ }^{15}$ Usually 1 to $2 \mathrm{mg} / \mathrm{kg}$ of lidocaine may be given as an intravenous bolus immediately prior to the ischemic event. Most effective neuroprotective techniques used today involve some combination of the above therapies associated with some level of hypothermia, which still seems to be the most effective for neural protection during periods of transient forebrain ischemia. ${ }^{28}$

\section{INTRAOPERATIVE MONITORING}

Intraoperative neurophysiological monitoring is used to improve postoperative outcome in patients undergoing skull base surgery, especially when cranial nerves are involved with tumor, are in the path of the surgical approach, or blood flow to brain tissue itself is at risk. In some cases nerve sacrifice is unavoidable and planned; however, because recovery of facial nerve function often occurs in two thirds of patients if the nerve is intact, it is the salvageable and potential inadvertent resection of cranial nerves that make EMG monitoring of nerves five, seven, nine, and ten most valuable. ${ }^{4}$ Meticulous surgical technique and intraoperative EMG monitoring have reduced the risk of facial nerve injury, especially in patients with tumors of the cerebellopontine angle and vestibular schwannomas. Current methods of facial nerve monitoring involve the recording of the specific muscles innervated by the facial nerve. This EMG activity is displayed on an oscilloscope with audible confirmation of muscle EMG activity. These monitoring systems will detect sev- 
eral types of physiological activity in the muscle, which are indicative of mechanical stimulation of the nerve or nerve trauma. Usually the administration of muscle relaxants is avoided during facial nerve monitoring but some investigators have performed low-dose infusions of muscle relaxants with good results; ${ }^{4}$ however, the use of muscle relaxants will reduce the ability to detect nerve irritation and quantify functional integrity. ${ }^{21}$ At our institution no muscle relaxant is administered after the initial intubating dose of an intermediate-acting muscle relaxant, usually atracurium. The halflife of this drug allows time for positioning and preparation of the patient, without movement prior to incision. The effects of the muscle relaxant are gone prior to the need to monitor the facial nerve. Depending on tumor size and involvement of the skull base, other cranial nerves could be monitored, which again would dictate minimal use of muscle relaxants.

Brainstem auditory evoked potentials and SSEPs may also be used during skull base surgery necessitating adjustment of the anesthetic technique to facilitate monitoring. In general, anesthetic effects on brainstem auditory evoked potentials are not dramatic with increasing concentrations of inhalational agents increasing waveform latencies. ${ }^{17}$ Body temperature will also change waveform characteristics, with reduced temperatures producing changes in the latency of the waveforms observed. Somatosensory evoked potential monitoring may also be used to detect ischemic injury or compression of the posterior fossa during skull base tumor resection. If an SSEP monitoring modality is to be used, the anesthetic technique must be altered to avoid high-dose inhalational anesthetic concentrations during surgery. This would cause a doserelated decrease in amplitude and increases in SSEP latency. ${ }^{23}$ Narcotic agents alter cortical SSEPs but changes are much less marked than with inhalational agents. Fentanyl will increase latency but produce minimal effects on waveform amplitude. Thus, an anesthetic technique involving the use of a low-dose inhalational agent (less than 1 minimum alveolar concentration.) and a narcotic infusion with $60 \% \mathrm{~N}_{2} \mathrm{O}$ in $\mathrm{O}_{2}$ may provide the most stable and optimal conditions for monitoring nerve function during skull base tumor resections.

\section{POSTOPERATIVE CARE}

After skull base surgery, the patients who have sufficiently recovered from anesthesia to follow commands are usually extubated. Presently, we extubate a large number of patients after prolonged procedures. Recovery from anesthesia is expedited by the use of short-acting, low solubility inhalational agents and infusions of potent narcotic agents. Respiratory parameters have to be adequate and the patient should be able to maintain blood $\mathrm{O}_{2}$ saturation while intubated and spontaneously breathing. As mentioned previously, excessive blood loss leading to large fluid and blood replacement could produce excessive airway edema, especially when the patient is prone. This would negate attempts at extubation. Cranial nerve deficits including injury to the vagus nerve producing vocal cord paralysis may occur after skull base surgery. Even though this would produce unilateral vocal cord paralysis, the patient should be awake and able to handle secretions so as to avoid the risk of aspiration. Once these variables are assessed and judged to be satisfactory, the patient is extubated.

Nausea and vomiting has been a frequent and important postoperative morbidity, which is of particular concern to the skull base surgical patient. Beyond the extreme discomfort, postoperative nausea and vomiting can lead to increased intracranial pressure, systemic hypertension, increased bleeding, worsening cerebrospinal fluid leaks, and an increased risk of aspiration, especially with postsurgical vocal cord dysfunction. ${ }^{24}$ Retrospective data from our surgical database indicate a high incidence of nausea after lateral approach skull base procedures (retrosigmoid and translabyrinthine) with $71 \%$ of patients complaining of either nausea or vomiting. Other approaches (suboccipital, craniofacial, and orbitocranial) produced a 54\% incidence of nausea and vomiting. Retrosigmoid craniotomies produced the highest incidence $(84 \%)$ and translabyrinthine approaches produced a $74 \%$ incidence of postoperative nausea and vomiting. Several methods have been used to reduce postoperative nausea. Ondansetron (4-8 mg intravenously) given 30 to 60 minutes before awakening from anesthesia has been of some benefit. ${ }^{12}$ Droperidol $(25 \mu \mathrm{g} /$ $\mathrm{kg}$ intravenously) has also been shown to be very effective as an antiemetic for procedures involving the osseous labyrinth of the ear; ${ }^{12}$ however, sedative effects coupled with a recent finding that droperidol causes QT wave prolongation and possibly torsades de pointes have made this a less appealing therapy for postoperative nausea and vomiting. ${ }^{14}$ Propofol has also been shown to have significant antiemetic properties. ${ }^{18}$ In our retrospective database, high-dose propofol anesthesia for skull base surgery was completely protective in reducing postoperative nausea and vomiting. Authors of some studies have demonstrated that subhypnotic infusions of propofol, started during the surgical procedure, are effective in reducing postoperative nausea and vomiting. ${ }^{22} \mathrm{We}$ are presently attempting to determine if this would be a beneficial therapy for patients having undergone skull base surgery.

The incidence, magnitude, and duration of acute pain experienced after skull base resection is not well known or characterized. Patients undergoing craniotomy have traditionally been thought to experience minimal postoperative pain; ${ }^{2}$ however, newer evidence suggests that neurosurgical patients receive inadequate analgesia from currently available regimens. In a preliminary study of craniotomy patients, De Benedittis, et al., ${ }^{6}$ reported that $60 \%$ of all patients who undergo craniotomy experience moderate to severe postoperative pain. Of these, $90 \%$ experienced pain in the first 12 hours after surgery, which lasted up to 48 hours. The pain that occurred was influenced by surgical route and was defined as pulsating or pounding, steady and sometimes stabbing in nature. In our study population, lateral approaches to the skull base required a lesser amount of narcotic over a 24-hour period than other skull base approaches (suboccipital and craniofacial). Although no ideal analgesic exists, we believe patient-controlled analgesia is extremely valuable after skull base surgical procedures. Patient-controlled analgesia has been found to be subjectively better for patients and leads to overall lower doses of opioid. ${ }^{29}$ Patient-controlled analgesia provides a mechanism to titrate drug administration, allows 
the patient to exert control over their pain management, and may alleviate the psychological stress aggravated by pain. Patient-controlled analgesia has been used for our patients who underwent skull base surgery for the past 2 years with no reported cases of respiratory depression and reintubation. We use morphine in a dosing regimen of 1.5 mg morphine/dose with an 8-minute lockout period. The total dose of morphine in 4 hours should not exceed 40 mg. Another common associated discomfort and a reason for dissatisfaction with patient-controlled analgesia is the development of postoperative nausea and vomiting. ${ }^{31}$ Previous studies have examined the use of antiemetics administered in combination with patient-controlled narcotics as a method to control nausea, vomiting, and pain. ${ }^{1}$ Several investigators have prescribed droperidol in combination with patient-controlled morphine. ${ }^{1,19}$ Although effective in reducing vomiting, droperidol increased the level of sedation. ${ }^{19}$ We have used a combination of patient-controlled morphine and ondansetron for postoperative pain and emesis. Preliminary comparisons with patient-controlled morphine therapy alone demonstrate reduced pain, nausea, and higher satisfaction with this combination patient-controlled therapy. ${ }^{11}$

\section{CONCLUSIONS}

The anesthesiologist is instrumental in providing optimum conditions for intraoperative monitoring while enabling the surgeon to accomplish the surgical procedure in a hemodynamically stable patient. The knowledgeable anesthesiologist can also improve postoperative outcomes and reduce morbidity provided he or she is given the information concerning surgical approach, tumor type, and involved vital structures. It is imperative that the neuroanesthesiologist discuss the procedure with the skull base surgical team and be involved in the decisions concerning perioperative management to produce an optimal outcome.

\section{References}

1. Alexander R, Lovell AT, Seingry D, et al: Comparison of ondansetron and droperidol in reducing postoperative nausea and vomiting associated with patient-controlled analgesia. Anaesthesia 50:1086-1088, 1995

2. Atkinson RS, Rushman GB, Davies NJH, et al: Lee's Synopsis of Anaesthesia, ed 11. Oxford, UK: Butterworth-Heineman, 1993

3. Busto R, Globus MY, Dietrich WD, et al: Effect of mild hypothermia on ischemia-induced release of neurotransmitters and free fatty acids in rat brain. Stroke 20:904-910, 1989

4. Cheek JC: Posterior fossa intraoperative monitoring. J Clin Neurophysiol 10:412-424, 1993

5. Cheney FW, Domino KB, Caplan RA, et al: Nerve injury associated with anesthesia: a closed claims analysis. Anesthesiology 90:1062-1069, 1999

6. De Benedittis G, Lorenzetti A, Migliore M, et al: Postoperative pain in neurosurgery: a pilot study in brain surgery. Neurosurgery 38:466-470, 1996

7. Duke DA, Lynch JJ, Harner SG, et al: Venous air embolism in sitting and supine patients undergoing vestibular schwannoma resections. Neurosurgery 42:1282-1287, 1998

8. Edelman GJ, Hoffman WE, Charbel FT: Cerebral hypoxic after etomidate administration and temporary cerebral artery occlusion. Anesth Analg 85:821-825, 1997
9. Edgington TS: Activation of the coagulation system in association with neoplasia. J Lab Clin Med 96: 1-4, 1980

10. Guggiari M, Lechat P, Gaven-Colonne C, et al: Early detection of patent foramen ovale by two-dimensional contrast echocardiography for prevention of paradoxical air embolism during sitting position. Anesth Analg 67:192-194, 1988

11. Hampson C, Moynihan G, Jellish WS, et al: PCA morphine and ondansetron for relief of post-operative pain, nausea, and vomiting in neurosurgical patients undergoing skull base procedures. Skull Base 12:15, 2002 (Abstract)

12. Jellish WS, Thalji Z, Fluter E, et al: Ondansetron versus droperidol or placebo when given prophylactically for the prevention of postoperative nausea and vomiting in patients undergoing middle ear procedures. J Clin Anesth 9:451-456, 1997

13. Kharasch ED, Sivarajan M: Gastroesophageal perforation after intraoperative transesophageal echocardiography. Anesthesiology 85:426-428, 1996

14. Lawrence KR, Nasraway SA: Conduction disturbances associated with administration of butyrophenone antipsychotics in critically ill: a review of the literature. Pharmacotherapy 17: 531-537, 1997

15. Lei B, Cottrell JE, Kass IS: Neuroprotective effect of low-dose lidocaine in a rat model of transient focal cerebral ischemia. Anesthesiology 95:445-451, 2001

16. Majasko J, Petrozza P, Cohen M, et al: Anesthesia and surgery in the seated position: analysis of 554 cases. Neurosurgery 17: 695-702, 1985

17. Manninen PH, Lam AM, Nicholas JF: The effects of isoflurane and isoflurane-nitrous oxide anesthesia on brainstem auditory evoked potentials in humans. Anesth Analg 64:43-47, 1985

18. McCollum JS, Milligan KR, Dundee JW: The antiemetic action of propofol. Anaesthesia 43:239-240, 1988

19. McKenzie R, Tantisira B, Jackson D, et al: Antiemetic efficacy of a droperidol-morphine combination in patient-controlled analgesia. J Clin Anesth 7:141-147, 1995

20. Michenfelder JD, Milde JH, Sundt TM Jr: Cerebral protection by barbiturate anesthesia. Use after middle cerebral artery occlusion in Java monkeys. Arch Neurol 33:345-350, 1976

21. Møller AR: Neurophysiological monitoring in cranial nerve surgery. Neurosurg Quart 5:55-72, 1995

22. Montgomery JE, Sutherland CJ, Kestin IG, et al: Infusions of subhypnotic doses of propofol for the prevention of postoperative nausea and vomiting. Anaesthesia 51:554-557, 1996

23. Peterson DO, Drummond JC, Todd MM: Effects of halothane, enflurane, isoflurane, and nitrous oxide on somatosensory evoked potentials in humans. Anesthesiology 65:35-40, 1986

24. Petrozza PH, Prough DS: Postoperative and intensive care, in Cottrell JE, Smith DS (eds): Anesthesia and Neurosurgery, ed 3. St Louis: Mosby, 1994, pp 625-659

25. Porter JM, Pidgeon C, Cunningham AJ: The sitting position in neurosurgery: a clinical appraisal. Br J Anaesth 82:117-128, 1999

26. Porter SS, Sanan A, Rengachary SS: Surgery and anesthesia of the posterior fossa, in Albin MA (ed): Textbook of Neuroanesthesia With Neurosurgical and Neuroscience Perspectives. St Louis: McGaw-Hill, 1997, pp 971-1008

27. Sakai T, Terao Y, Miyata S, et al: [Postoperative recurrent laryngeal nerve palsy following a transesophageal echocardiography.] Masui 48:656-657, 1999 (Jpn)

28. Schmid-Elsaesser R, Hungerhuber E, Zausinger S, et al: Combination drug therapy and mild hypothermia: a promising treatment strategy for reversible, focal cerebral ischemia. Stroke 30:1891-1899, 1999

29. Smythe MA, Martin SE, Schad RF: Retrospective evaluation of patient-controlled analgesia use in a large teaching institution. DICP 25:1058-1061, 1991

30. Tsuda H, Oka K, Noutsuka Y, et al: Tissue-type plasminogen activator in patients with intracranial meningiomas. Thromb Haemost 60:508-513, 1988 


\section{Anesthesiology in complex skull base surgery}

31. Tsui SL, Tong WN, Irwin M, et al: The efficacy, applicability and side-effects of postoperative intravenous patient-controlled morphine analgesia: an audit of 1233 Chinese patients. Anaesth Intensive Care 24:658-664, 1996

32. Tu YK, Heros RC, Candia G, et al: Isovolemic hemodilution in experimental focal cerebral ischemia. Part I: Effects on hemodynamics, hemorheology, and intracranial pressure. J Neurosurg 69:72-81, 1988

33. Voll CL, Auer RN: Insulin attenuates ischemic brain damage independent of its hypoglycemic effect. J Cereb Blood Flow Metab 11:1006-1014, 1991

34. von Gosseln H, Samii M, Suhr D, et al: The lounging position for posterior fossa surgery: anesthesiological considerations regarding air embolism. Childs Nerv Syst 7:368-374, 1991

35. Waltz AG: Effect of blood pressure on blood flow in ischemic and nonischemic cerebral cortex. The phenomena of autoregulation and luxury perfusion. Neurology 18:613-621, 1968

36. Wass C, Scheithauer B, Bronk J, et al: Insulin treatment of corticosteroid-associated hyperglycemia and its effect on outcome after forebrain ischemia in rats. Anesthesiology 84:644-651, 1996
37. Wass CT, Lanier WL: Glucose modulation of ischemic brain injury: review of clinical recommendations. Mayo Clin Proc 71: 801-812, 1996

38. Wass CT, Lanier WL: Hypothermia-associated protection from ischemic brain injury: implication for patient management. Int Anesthesiol Clin 34:95-111, 1996

39. Wiet RJ, Teixido M, Liang JG: Complications in acoustic neuroma surgery. Otolarygol Clin North Am 25:389-412, 1992

40. Zhu H, Cottrell JE, Kass IS: The effect of thiopental and propofol on NMDA- and AMDA-mediated glutamate excitotoxicity. Anesthesiology 87:944-951, 1997

Manuscript received March 18, 2002.

Accepted in final form April 22, 2002.

Address reprint requests to: W. Scott Jellish, M.D., Ph.D., Department of Anesthesiology, Loyola University Medical Center, 2160 South First Avenue, Maywood, Illinois 60153. email: wjellis @1umc.edu. 